\title{
Effect of Tissue Elasticity in Cardiac Radiofrequency Catheter Ablation Models
}

\author{
Argyrios Petras $^{1}$, Massimiliano Leoni ${ }^{1,2}$, Jose M Guerra ${ }^{3}$, Johan Jansson ${ }^{1,2}$, Luca Gerardo-Giorda ${ }^{1}$ \\ ${ }^{1}$ Basque Center for Applied Mathematics, Bilbao, Spain \\ ${ }^{2}$ KTH Royal Institute of Technology, Stockholm, Sweden \\ ${ }^{3}$ Hospital de la Santa Creu i San Pau, Barcelona, Spain
}

\begin{abstract}
Radiofrequency catheter ablation (RFCA) is an effective treatment for different types of cardiac arrhythmias. However, major complications can occur, including thrombus formation and steam pops. We present a full $3 D$ mathematical model for the radiofrequency ablation process that uses an open-irrigated catheter and accounts for the tissue deformation, an aspect overlooked by the existing literature. An axisymmetric Boussinesq solution for spherical punch is used to model the deformation of the tissue due to the pressure of the catheter tip at the tissue-catheter contact point. We compare the effect of the tissue deformation in the RFCA model against the use of a standard sharp insertion of the catheter in the tissue that other state-ofthe-art RFCA computational models use.
\end{abstract}

\section{Introduction}

Radiofrequency catheter ablation is a minimally invasive, effective treatment for various types of cardiac arrhythmias. Typically, for endocardiac RFCA, a catheter is inserted in the cardiac chamber and placed at the arrhythmogenic tissue. Resistive electrical heating is produced in a neighborhood of the electrode, which propagates by conduction to the rest of the tissue, causing irreversible damage to the latter and the formation of a lesion at $50{ }^{\circ} \mathrm{C}$ [1]. Radiofrequency ablation (RFA) is an effective and a safe procedure for cardiac arrhythmias; however a number of life-threatening complications can occur, including the possibility of thrombus formation, in case the blood temperature rises above $80^{\circ} \mathrm{C}$, and steam pops in the occurrence of tissue overheating (around $100{ }^{\circ} \mathrm{C}$ ) [1].

Several computational models have been introduced to describe the biophysics of RFA in two and three dimensions $[2,3]$. Typically, a modified Penne's bioheat equation is considered for the temperature changes, while a quasistatic equation describes the spatial distribution of the electrical potential. Some models include the blood flow either as boundary conditions for the bioheat equation in the tissue or using the Navier-Stokes equations. However, the state-of-the-art models overlook the mechanical deformation of the tissue due to the contact with the catheter. Until recently, only [4] used profiles extracted from X-ray scanning to identify the deformation of the tissue; however this approach is partial and dependent on the tissue specimen. We introduced in [5] a methodological approach, using a solution of the axisymmetric Boussinesq problem for a spherical punch. The model has been validated against experiments designed ad-hoc [5]. We explore here the effect of the tissue elastic deformation against the sharp insertion (undeformed case) currently used from state-of-the-art models in the RFA modelling.

\section{Mathematical Model}

\subsection{Computational geometry}

A box of size $80 \mathrm{~mm} \times 80 \mathrm{~mm} \times 80 \mathrm{~mm}$ is considered, that consists of five different components: the blood chamber $80 \mathrm{~mm} \times 80 \mathrm{~mm} \times 40 \mathrm{~mm}$, the cardiac tissue 80 $\mathrm{mm} \times 80 \mathrm{~mm} \times 20 \mathrm{~mm}$, a board $80 \mathrm{~mm} \times 80 \mathrm{~mm} \times 20$ $\mathrm{mm}$ that models the external effects between the tissue and the dispersive electrode, the electrode and the thermistor. The electrode is placed at the center of the blood chamber, has $3.5 \mathrm{~mm}$ length and $2.33 \mathrm{~mm}$ diameter, contains 6 irrigation holes of diameter $0.5 \mathrm{~mm}$ and has a hemispherical tip of radius $1.165 \mathrm{~mm}$. The thermistor is placed inside the electrode and has a diameter of $1.54 \mathrm{~mm}$ and a length of $3 \mathrm{~mm}$. Figure 1 shows the geometry considered.

\subsection{Governing equations}

During the RFA treatment, the catheter is placed on the arrhythmogenic tissue and causes the deformation of the latter. Using an axisymmetric Boussinesq solution for a spherical indenter in contact mechanics [6], the vertical deformation of the tissue is described as 

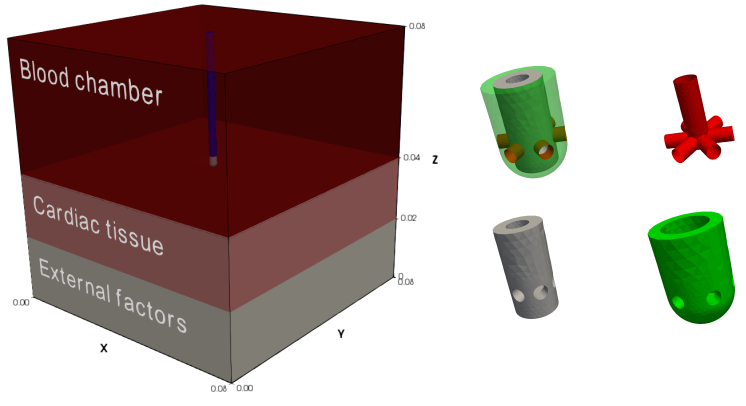

Figure 1. Left: The full computational geometry. Right: The computational tip of the catheter (top left), the saline tubes (top right), the thermistor (bottom left) and the electrode (bottom right).

$\Delta z(r)= \begin{cases}\omega_{\max }-\left(R-\sqrt{R^{2}-r^{2}}\right), & r \leq a, \\ \frac{a^{2}}{\pi} \int_{0}^{1} \frac{2 \omega_{\max }-a t \log \left(\frac{R+a t}{R-a t}\right)}{r^{2}-a^{2} t^{2}} d t, & r>a,\end{cases}$

where $R$ is the radius of the hemispherical tip of the electrode, $r$ is the distance from the center of the electrode, $\omega_{\max }$ is the maximum deformation (which occurs at the center of the electrode) and $a$ is the contact radius of the electrode and the tissue. The maximum depth and the contact radius can be calculated using the contact force $F$ as

$$
\begin{gathered}
F=\frac{G}{1-\nu}\left(\left(a^{2}+R^{2}\right) \log \left(\frac{R+a}{R-a}\right)-2 a R\right), \\
\omega_{\max }=\frac{a}{2} \log \left(\frac{R+a}{R-a}\right),
\end{gathered}
$$

where $G$ is the shear modulus and $\nu$ is the Poisson's ratio of the tissue. The elastic deformation $\Delta z$ is applied to the cardiac tissue in the computational geometry.

The catheter is surrounded by blood and continuously flushes saline through the irrigation holes on the electrode at its tip to cool the ablation site. The incompressible Navier-Stokes equations are employed to model the bloodsaline interaction. A constant inflow is considered on a side of the blood chamber, with the corresponding outflow boundary conditions on the opposite side; a radial inflow towards the blood is considered from the saline tubes. All the remaining boundaries, including the blood-tissue interface, are equipped with no slip conditions.

During the ablation, electrical current flows through the catheter to the tissue, causing Joule heating. A modified Penne's bioheat equation is typically used to describe the temperature change [3] $\rho c(T)\left(\frac{\partial T}{\partial t}+\mathbf{v} \cdot \nabla T\right)-\nabla \cdot(k(T) \nabla T)=q+Q_{m}-Q_{p}$,

where $T$ is the temperature, $\mathbf{v}$ is the velocity from the Navier-Stokes equations, $\rho$ is the density, $c$ is the specific heat, $k$ is the thermal conductivity, $Q_{m}$ is the metabolic heat, $Q_{p}$ is the heat loss from blood perfusion and $q$ is the heat source. For endocardiac RFA, the metabolic and blood perfusion heat $Q_{m}$ and $Q_{p}$ are negligible and thus omitted in our model [3].

The heat source produced by the electrical potential is $q=\sigma(T)|\nabla \Phi|^{2}$, where $\sigma$ is the electrical conductivity and $\Phi$ is the electrical potential. For current at frequencies of $500 \mathrm{kHz}$ and over the distance of interest, the system is considered totally resistive, thus a quasi-static equation can be employed to model the potential distribution

$$
\nabla \cdot(\sigma(T) \nabla \Phi)=0 .
$$

For constant power ablation, the quasi-static equation is augmented with a constraint

$$
\int_{\Omega} \sigma(T)|\nabla \Phi|^{2} d x=P,
$$

where $\Omega$ is the computational domain and $P$ is the total power dissipated in the system.

Regarding the boundary conditions of the above equations, the body temperature of $37^{\circ} \mathrm{C}$ is applied on all outer boundaries of the computational geometry. The temperature on the irrigation holes is set to $22^{\circ} \mathrm{C}$, and insulation boundary conditions are applied on the interfaces shared with the catheter body and on the saline pipes (shown in red in Figure 1). In terms of the boundary conditions for the electrical potential, a constant voltage $V_{0}$ is imposed on the catheter-electrode interface (the top surface of the green electrode in Figure 1) calculated to satisfy the power constraint equation. Zero potential is enforced at the bottom of the computational geometry, while zero flux conditions are imposed in all the remaining boundaries.

\subsection{Power distribution}

The machines used in the RFA treatment provide the impedance of the full system during the ablation. In our computational model, the electrical conductivity of the external effects board (see Figure 1) is tuned to match the initial impedance provided by the RFA machine. In particular, for a constant power ablation, the initial $V_{0}$ can be identified by Ohm's law. By enforcing $V_{0}$ in the quasistatic electrical potential equation, and by enforcing a constraint

$$
\int_{\Omega_{t}} \sigma(T)|\nabla \Phi|^{2} d x=P_{t}
$$


where $\Omega_{t}$ is the cardiac tissue and $P_{t}$ is the power delivered to the tissue, optimization techniques are used to identify the electrical conductivity of the board [5]. The quantity $P_{t}$ depends on the tissue electrical conductivity and the tissueelectrode contact surface area, and can be calculated using the technique described by Wittkampf et al. [7]. In particular,

$$
P_{t}=\frac{A_{t} \sigma_{t}}{A_{b} \sigma_{b}+A_{t} \sigma_{t}} P_{\text {nom }}=: \alpha P_{\text {nom }},
$$

where $A_{t}$ and $A_{b}$ are the surface area of the electrode in contact with the tissue and the blood respectively, $\sigma_{t}$ and $\sigma_{b}$ are the tissue and the blood electrical conductivities and $P_{n o m}$ is the nominal power of the ablation protocol. The electrical conductivity of the board remains constant throughout the RFA simulation.

Due to the scaling and other external factors, the total power from the RFA machine cannot coincide with the power dissipated within our computational geometry. Thus, the total power dissipated in our computational system $P$ is calculated in a preprocessing step as

$$
\int_{\Omega} \sigma(T)|\nabla \Phi|^{2} d x=P .
$$

This power $P$ is kept constant throughout the RFA simulation, and corresponds to the constraint equation imposed on the electrical potential quasi-static equation.

\section{Results}

In our computational simulations, the open source software Salome [8] is used for the geometry generation, FEniCS-HPC [9] for the numerical solution of our model using the finite element method and Paraview [10] for the postprocessing of the computational lesion and the visualizations.

\subsection{Parameters}

The parameters used in the mathematical model are collected from the literature. In particular, the physical parameters of the electrode and the thermistor can be found in [3]. The blood physical parameters are found in the virtual population database from the IT IS foundation [11]. A porcine cardiac tissue is considered with electrical properties as appear in [2] and thermal properties as described in [12]. In particular, a linear decrease of the specific heat with respect to temperature of $0.42 \%$ from the value $3017 \mathrm{~J} \mathrm{~kg}^{-1} \mathrm{~K}^{-1}$ at body temperature is considered. Similarly, for the thermal conductivity a decrease of $0.05 \%$ from the value $0.518 \mathrm{~W} \mathrm{~m}^{-1} \mathrm{~K}^{-1}$ at body temperature, and for the electrical conductivity an increase of $1.5 \%$ from the value $0.54 \mathrm{~S} \mathrm{~m}^{-1}$ at body temperature. Regarding the mechanical properties of the cardiac tissue, the Poisson's ratio is chosen as 0.499 and the shear modulus as $25 \mathrm{kPa}$ [13]. Finally, the physical parameters of the external effects board are chosen to be constant, as the corresponding ones of the cardiac tissue at body temperature.

\subsection{Discussion}

In our numerical simulations, a constant power ablation protocol is considered of $20 \mathrm{~W}$ for $30 \mathrm{~s}$. The saline inlet is set as $17 \mathrm{~mL} / \mathrm{min}$, following the instructions of the catheter producer. Three different contact forces are considered: $10 \mathrm{~g}, 20 \mathrm{~g}$ and $40 \mathrm{~g}$. In the undeformed tissue case, the catheter is placed at the same depth as the one calculated for the deformed case. The power delivery is controlled according to the procedure described in the previous section for both the elastic and sharp insertions. Two blood flow velocity profiles are considered that are commonly used in experiments: a high blood flow of $0.5 \mathrm{~m} \mathrm{~s}^{-1}$ and a low blood flow of $0.1 \mathrm{~m} \mathrm{~s}^{-1}$. The computational lesions are identified by tracking the $50{ }^{\circ} \mathrm{C}$ isotherm contour. Figure 2 shows the numerical results for the case of high blood flow, for different contact forces. In case of steam pop occurrence at $100{ }^{\circ} \mathrm{C}$, the lesion is shown right at the popping time.

In the case of $10 \mathrm{~g}$ and high blood flow, a small lesion is formed in the elastic case after the completion of $30 \mathrm{~s}$, while the temperature in the sharp insertion profile case reaches $100{ }^{\circ} \mathrm{C}$ after $21.6 \mathrm{~s}$, which hints for steam pop formation. Similarly, in the cases of $20 \mathrm{~g}$ and $40 \mathrm{~g}$, the elastic case provides a lesion with maximum tissue temperature of $77^{\circ} \mathrm{C}$ and $93^{\circ} \mathrm{C}$ respectively, while steam pops occur in the sharp case after the completion of $5.4 \mathrm{~s}$ and $2.5 \mathrm{~s}$. Similar observations can be drawn for the results of the low blood flow.

\section{Conclusions}

The elastic deformation of the tissue is an important factor in the RFA modelling, which allows the calculation of the force-insertion depth relation as well as the power dissipated in the tissue. In addition, the elastic insertion allows for a more realistic and accurate representation of the cardiac tissue. Finally, our computational results show that the sharp insertion overestimates the tissue temperature rise, hinting for steam pop formation even at low contact force and power ablation protocols.

\section{Acknowledgements}

This research was supported by the Basque Government through the BERC 2014-2017 and BERC 2018-2021 program and by Spanish Ministry of Economy and Competitiveness MINECO through BCAM Severo Ochoa excellence accreditations SEV-2013-0323 and SEV-2017-0718, 

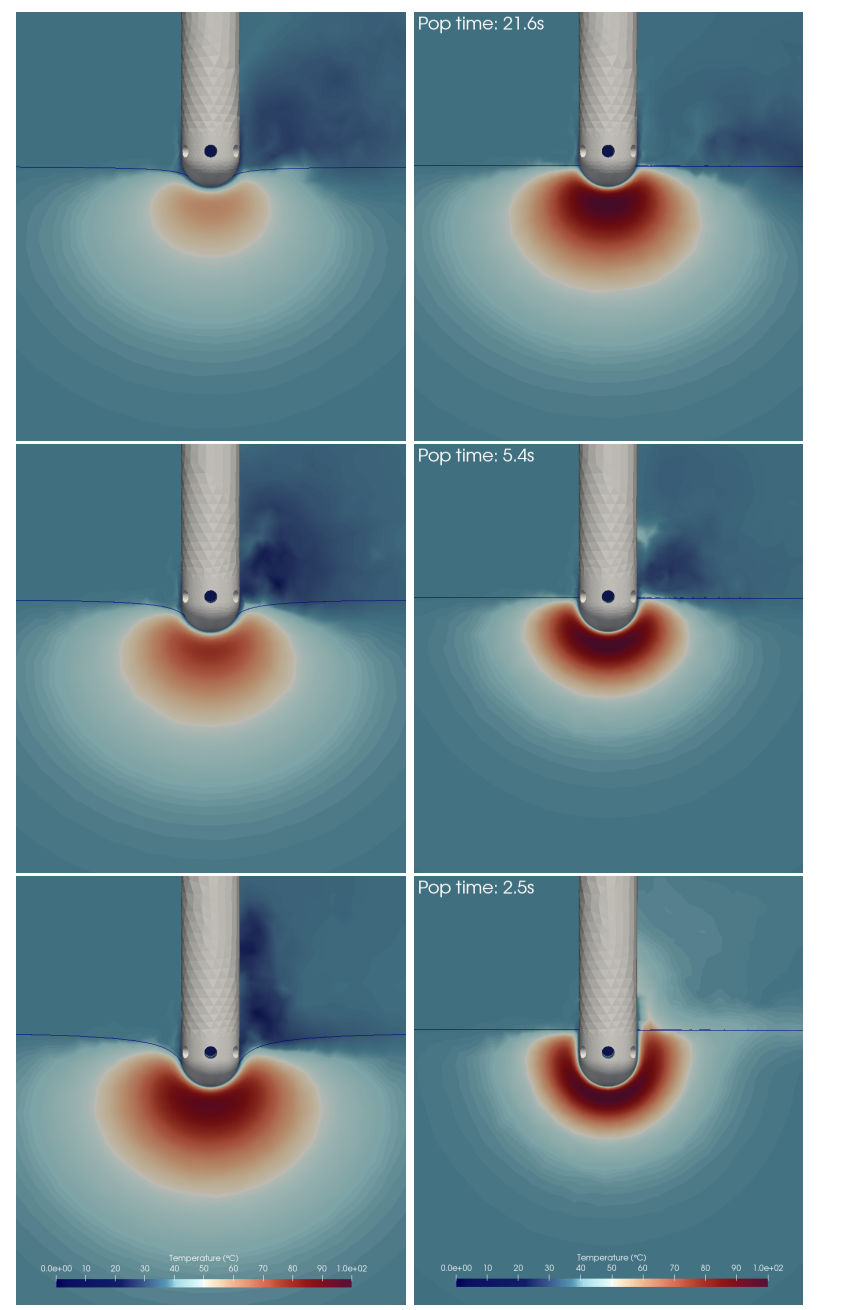

Figure 2. The computational lesions for high blood flow using the elastic insertion (left) and the sharp insertion (right) for $10 \mathrm{~g}, 20 \mathrm{~g}$ and $40 \mathrm{~g}$ (top to bottom).

and through projects MTM2015-69992-R and MTM201676016-R. JJ acknowledges support from project EU H2020 MSO4SC. ML acknowledges the "LaCaixa 2016" PhD Grant.

\section{References}

[1] Huang SKS, Wood MA. Catheter Ablation of Cardiac Arrhythmias E-book. Elsevier Health Sciences, 2014.

[2] Berjano EJ. Theoretical modeling for radiofrequency ablation: state-of-the-art and challenges for the future. Biomedical Engineering Online 2006;5(1):24.

[3] González-Suárez A, Berjano E, Guerra JM, Gerardo-Giorda L. Computational modeling of open-irrigated electrodes for radiofrequency cardiac ablation including blood motionsaline flow interaction. PloS One 2016;11(3):e0150356.

[4] Cao H, Speidel MA, Tsai JZ, Van Lysel MS, Vorperian VR, Webster JG. Fem analysis of predicting electrode- myocardium contact from rf cardiac catheter ablation system impedance. IEEE Transactions on Biomedical Engineering 2002;49(6):520-526.

[5] Petras A, Leoni M, Guerra JM, Jansson J, Gerardo-Giorda L. A computational model of open-irrigated radiofrequency ablation including the mechanical properties of the cardiac tissue. International Journal for Numerical Methods in Biomedical Engineering 2018; (submitted).

[6] Sneddon IN. The relation between load and penetration in the axisymmetric boussinesq problem for a punch of arbitrary profile. International Journal of Engineering Science 1965;3(1):47-57.

[7] Wittkampf FH, Nakagawa H. Rf catheter ablation: Lessons on lesions. Pacing and Clinical Electrophysiology 2006; 29(11):1285-1297.

[8] Ribes A, Caremoli C. Salome platform component model for numerical simulation. In Computer Software and Applications Conference, 2007. COMPSAC 2007. 31st Annual International, volume 2. IEEE, 2007; 553-564.

[9] Hoffman J, Jansson J, Jansson N. Fenics-hpc: Automated predictive high-performance finite element computing with applications in aerodynamics. In International Conference on Parallel Processing and Applied Mathematics. Springer, 2015; 356-365.

[10] Ayachit U. The paraview guide: a parallel visualization application. Kitware, Inc., 2015.

[11] Hasgall P, Neufeld E, Gosselin M, Klingenböck A, Kuster N. Itis database for thermal and electromagnetic parameters of biological tissues. Version 30 2015;

[12] Bhavaraju N, Valvano J. Thermophysical properties of swine myocardium. International Journal of Thermophysics 1999;20(2):665-676.

[13] Urban MW, Pislaru C, Nenadic IZ, Kinnick RR, Greenleaf JF. Measurement of viscoelastic properties of in vivo swine myocardium using lamb wave dispersion ultrasound vibrometry (lduv). IEEE Transactions on Medical Imaging 2013;32(2):247-261.

Address for correspondence:

Argyrios Petras

BCAM - Basque Center for Applied Mathematics

Mazarredo 14, 48009, Bilbao, Basque Country, Spain

apetras@bcamath.org 